\title{
Powder flow behavior governed by the surface properties of glass beads
}

\author{
Shirin Enferad ${ }^{\mathrm{a}, \mathrm{b}, *}$, Salvatore Pillitteri $^{\mathrm{c}}$, Geoffroy Lumay ${ }^{\mathrm{c}}$, Claire Gaiani ${ }^{\mathrm{b}}$, \\ Sebastien Kiesgen De Richter ${ }^{a}$, Michaël Marck ${ }^{c}$, Syrym Umbetov ${ }^{\mathrm{a}}$, Nicolas Vandewalle ${ }^{\mathrm{c}}$, Mathieu Jenny ${ }^{\mathrm{a}}$ \\ a Université de Lorraine, Laboratoire d'Energétique et de Mécanique Théorique et Appliqué (LEMTA), CNRS, UMR 7563, Vandoeuvre-lés-Nancy F-54500, France \\ b Université de Lorraine, Laboratoire d'Ingénierie des Biomolécules (LIBio), 2 avenue de la Forêt de Haye, TSA 40602, 54518 Vandoeuvre-lès-Nancy, France \\ c GRASP, Institute of Physics B5a, University of Liège, B4000 Liège, Belgium
}

\section{A R T I C L E I N F O}

\section{Article history:}

Received 8 March 2021

Received in revised form 29 April 2021

Accepted 30 April 2021

Available online 4 May 2021

\section{Keywords:}

Flowability

Glass bead

Hydrophobic formulation

Humid control

\begin{abstract}
A B S T R A C T
Powder rheology and its sensitivity to surrounding environmental condition by controlling the surface properties of the particles is one of the major challenges of the powder industries. Indeed, handling large quantities needs powders with good flowability, adequate compressibility and few electrostatic charges. We have performed a chemical treatment in order to obtain hydrophobic glass beads and its bulk behavior has been compared with raw glass beads depending on the humidity. We characterized flow properties under different processing equipments. We observed that by performing hydrophobic surface treatment sensitivity of glass beads reduced to the humidity. Furthermore, the influence of the electrostatic charges was an undeniable factor in increasing the viscosity of hydrophobic glass beads and consequently lowering its flowability in front of raw glass beads; at low shear rate. At high shear rate, the powders presented similar behaviors.
\end{abstract}

(c) 2021 Elsevier B.V. All rights reserved.

\section{Introduction}

Powders are omnipresent in our daily life and they are extensively used in various industries such as pharmaceutical, cosmetics, chemical, food and construction. Despite numerous studies on powder rheology [1-7] their behavior is not fully understood, due to their complexity. In fact, the flow behavior of a powder depends on many factors including surface properties, size, shape of particles [8] and environmental conditions such as humidity [9].

However, understanding the powder's flowability is one the most important objectives in powder industries. Indeed, it can allow them to anticipate the powder's characteristics by knowing the condition that they encounter during process. In order to identify the pertinent parameters governing the powder's flow, using different test methods that represent the state of stress and strain rate of interest is very important. These methods can provide different points of view and indices to assess powder flowability with several mechanical and dynamical conditions that have been explained in the following.

\footnotetext{
* Corresponding author at: Université de Lorraine, Laboratoire d'Energétique et de Mécanique Théorique et Appliqué (LEMTA), CNRS, UMR 7563, Vandoeuvre-lés-Nancy F54500, France \& Université de Lorraine, Laboratoire d'Ingénierie des Biomolécules (LIBio), 2 avenue de la Forêt de Haye, TSA 40602, 54518 Vandoeuvre-lès-Nancy, France. E-mail addresses: shirin.enferad@univ-lorraine.fr, shirinenferad@yahoo.com (S. Enferad).
}

It is known that the Hausner ratio is an efficient and rapid method to estimate the powder behavior based on bulk density [10], Hausner ratio correlated to the ratio between settled bulk density and tapped bulk density. It has been reported that low compressible powders have the best flowability [11]. Similarly, a study on copper powder indicated that the Hausner ratio can be representative of friction condition between particles [12].

Apparition of powder electrostatic charges as a result of particleparticle interaction and powders interaction with manipulating equipment during powder process is a know phenomena in industries. In addition, depending on the different surface chemical formulation of powders, the electrostatic charge can increase or decrease which impacts the powder bulk behavior [13,14]. Whereby, an increase in powder electrostatic charge can lower its flowability $[15,16]$. Powder characterization based on their electrostatic charge is of great interest [17-19].

Moreover, rotating drum is a method to characterize the macroscopic properties of powders under aeration condition, it fluidizes the powder and reports evolution of cohesion under rotation [6]; this technique measures powder flow at high shear rate condition. Furthermore, one of the most used methods in industry is applying vibration during process. This technique optimizes powder flowability consequently enhances saving energy. In addition, this method is convenient to control the different steps of industrial process consisting of feeding, conveying and even parts of the equipment such as hopper, etc. [20,21]. The mentioned technique has been studied recently by implementing a 
rheometer attached to a shaker [21]. Based on vibrational rheology, the powder flow can be assessed at low shear rate condition.

On the other side, studying and understanding the influence of environmental condition like as humidity on powder behavior is very significant and of great interest. A large number of theoretical and experimental studies in the literature are devoted to figuring out the link between air humidity and powder flow behavior [22-30]. A decrease in flowability with increasing relative air humidity has been reported for granular material $[26,27,29]$ and food powders $[25,30]$.

This paper focuses on studying the influence of surface treatment on powder flowability and sensitivity of powder flowability to the external condition. Therefore, first in section III.A, the influence of surface treatment on the behavior of raw and hydrophobic glass beads has been studied in ambient temperature without humid control. Different methods utilized to figuring out the macroscopic flow and the rheology of powders under different processing dynamics. Then in section III.B, the influence of humidity on powder behavior has been studied. In this case, two different methods of humid control and powder rheology utilized to characterize the powders and finally their results have been compared.

First, the humid control was done inside a rotating drum with humid-air controlled flow. The powders were kept for $1 \mathrm{~h}$ in each selected humid range.

A second experiment with a different humid control method was conducted at the same time, in order to compare and to link the dependency of the humidity on the shear rate with the electrostatic charge of powders and their cohesion.

In this case, the powders were kept in a humid chamber for $72 \mathrm{~h}$ at $20{ }^{\circ} \mathrm{C}$ in a given humidity ranging from $35 \%$ up to $90 \% \mathrm{RH}$. The humid control time kept long enough to be sure that the powders took enough humidity at each humid value and at the end of humid control the humidity value of powders were confirmed by TH200 thermoconstanter. Then the rheology of powders was performed in a dry room in ambient temperature during maximum 30 min of measurement. The apparent viscosity of powders has been measured with Discovery HR3Rheometer. The rheometer was attached to a shaker in order to ensure a reproducible packing state and collect data at low shear rate condition. All results have been collected by imposing shear stress.

\section{Materials and methods}

In the following, the implemented materials and the different methods for characterizing powders have been explained. The purpose of using different methods is to characterize powders under different processing dynamics and to observe the influence of treatment and mainly the effect of humidity on powders flowability. Therefore, two series of tests have been performed on raw and hydrophobic glass beads. First, the characterization measurements have been done by implementing the Granutools equipments. The packing dynamics of powders has been measured with the GranuPack, the powder electrostatics charge studied with the GranuCharge and the powder flowability and cohesion measured with GranuDrum equipment. During performing measurements with the Granutools equipments, the humidity and temperature were kept constant at $37-44 \% \mathrm{RH}$ and $22^{\circ} \mathrm{C}$, respectively. All Granutools measurements have been repeated three times.

Then the study of influence of wide range of humidity has been done on powders with the GranuDrum and the GranuCharge. These results have been confronted with the measurements of the powder behavior (after longer humid control) under vibrational rheology with Discovery HR3-Rheometer which provides possibility of powder flow at low shear rate where the cohesion of powders play a role in their flowability. The goal here was to observe clearly the effect of humidity on powders behaviors based on the strength of their surface cohesion depending on their surface treatment.

\subsection{Powders preparation and conditioning}

In this study, glass beads of type S 90-150 $\mu$ m have been used. Hydrophobic surface treatment has been done on the glass beads. With the purpose of preparing hydrophobic surface treatment on the glass beads [31,32], first the hydrophilic surface treatment has been prepared. The hydrophilic treatment [31] was achieved with a 3:1 mixture of sulfuric acid and hydrogen peroxide. First, $50 \mathrm{~g}$ of glass beads was put in a beaker and immersed in sulfuric acid then hydrogen peroxide was gently added. The mixture was kept $4 \mathrm{~h}$ at ambient temperature (20 ${ }^{\circ} \mathrm{C}$ ) under extractor hood. After that, the glass beads was washed with distilled water then silanization protocol has been followed. The silanization of glass beads with mixture of toluene $(500 \mathrm{~mL})$ and $1 \mathrm{H}, 1 \mathrm{H}, 2 \mathrm{H}, 2 \mathrm{H}$-perfluorooctyltriethoxysilane $(2.5 \mathrm{~g})$ has been performed; this mixture has been kept maximum $72 \mathrm{~h}$ under extractor hood at ambient temperature and then the powder was filtered and washed with pure toluene and finally was kept about $72 \mathrm{~h}$ under extractor hood for evaporating the toluene inside the powder. At the last, with the purpose of completely dry the powder, it was kept in an oven, maximum 4 h at $70^{\circ} \mathrm{C}$.

By performing contact angle measurement, the wetting behavior of hydrophobic glass beads has been measured to verify whether the surface of glass beads was chemically modified. A mono-layer of hydrophobic glass beads has been prepared on a microscope slide then a droplet of water has been put over the sample. The contact angle has been measured based on taken images from sample and five replicates were performed for each sample, under ambient conditions. The contact angle for hydrophobic glass beads was $142^{\circ} \pm 3^{\circ}$. This value was larger than $90^{\circ}$ which is a confirmation of hydrophobic surface formulation.

\subsection{GranuDrum/rheometer}

The GranuDrum is one of the most extensively used practical geometry to study the flow behavior of granular material [6,33-35]; especially non cohesive powders [6]. GranuDrum evaluates the flow properties of powders in a free flowing regime and the only applied stress on the powders is due to their own weight [36,37]. The experimental set up in this study presented in Fig. 1, consists of a drum with two glass walls which rotates around its central axis with the angular velocity $\Omega$. The flowing dynamics of powders inside the drum is function of this rotating angular velocity. The drum is generally half-filled with the powder whereas the length and the diameter of the drum are respectively $20 \mathrm{~mm}$ and $84 \mathrm{~mm}$. During rotation, the drum is back lighted by using a stroboscope and a CCD camera takes pictures of powder-air interface position inside drum. For each imposed angular velocity, 50 pictures are taken, separated by $0.5 \mathrm{~s}$. After monitoring the flow of powders via a camera then the images are processed by using image-processing algorithm. In the images, the granular material appears in black and the air appears in white, whereby an edge detection determines the position of the powder-air interface. From this analysis, two measurements are extracted: the average interface position and the fluctuations around this average position. Both of these

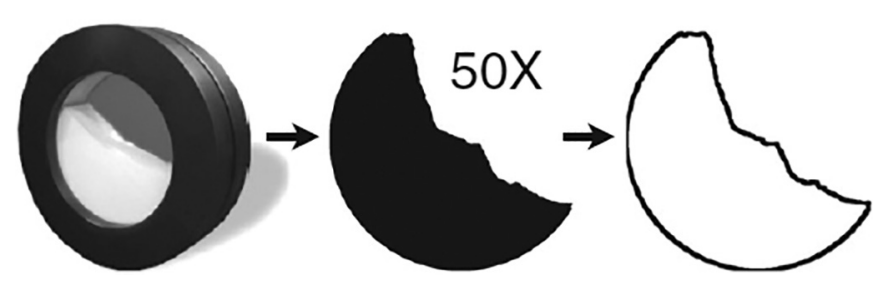

Fig. 1. Sketch of the GranuDrum equipment [38]. A cylinder is half-filled with the powder and rotates around its axis. For each rotating speed, a CCD camera takes 50 successive images of the flow. A dedicated algorithm enables to measure the flowing angle $\alpha_{f}$ and the fluctuations $\sigma_{f}$ of the flow from the shape of the powder-air interface. 
measurements are calculated according to the average of 50 pictures that have been taken from powder-air interface inside rotating drum in each imposed angular velocity. Based on the average interface position, the flowing angle $\alpha_{f}$ is measured in the center of the flow which is the angle between the horizontal and the average interface. Also, the standard deviation calculated from the fluctuations of the powderair interface, this parameter is called cohesive index $\sigma_{f}$ and is directly linked to the cohesion inside drum. In fact, in the range of considered rotating speed, the cohesive granular material leads to irregular flow and non-cohesive granular material leads to a continuous flow. In this study, the rotating angular velocity has been selected between 2 and $30 \mathrm{rpm}$.

\subsection{GranuPack}

Measuring the tapped density and packing dynamic of powders is very popular in powder characterization $[6,10]$. The GranuPack equipment presented in Fig. 2 developed to automate the procedure since it is usually realized with naked eyes. The analyzing cell is made of a tube of $D=26 \mathrm{~mm}$ in diameter and $L=150 \mathrm{~mm}$ in length. This tube is metallic to avoid the accumulation of electrostatic charges during measurement. A narrower and bottomless tube $\left(L=170 \mathrm{~mm}, D_{\text {inner }}=\right.$ $22.2 \mathrm{~mm}$ and $D_{\text {outer }}=25.4 \mathrm{~mm}$ ) is inserted into this measurement tube and filled with the powder to analyze. Afterward, it is slowly removed upward at the velocity $v=1 \mathrm{~mm} / \mathrm{s}$. This initialization procedure avoids human intervention and then increases the reproducibility.

A light hollow cylinder is placed on the top of the pile to keep it flat during the compaction process. The tube goes up and down over a height of $\Delta z=3 \mathrm{~mm}$ with the frequency $f=1000 \mathrm{~ms}$ in order to compact the granular pile. A distance sensor measures position of the hollow cylinder after each tap and computes the bulk volume $V$ of the pile. As the introduced mass of powder is known, then the evolution of bulk density of powder $\rho_{b}$ has been calculated as a function of tap number $t$. The bulk density is the ratio between mass $m$ and volume $V$ of the powder. The packing fraction $\eta$ is calculated by dividing bulk density $\rho_{b}$ by the true density of particles $\rho_{t}$. After recording packing fraction for each tap, compaction curve has been fitted with the classical logarithmic model [39],

$\eta(t)=\eta_{\infty}-\frac{\eta_{\infty}-\eta_{0}}{1+\ln (1+t / \tau)}$

where, $\mathrm{t}$ is the number of tapes, the fitting parameters $\eta_{\infty} \& \tau$ are asymptotic volume fraction and compaction characteristic time, respectively. The asymptotic volume fraction is representative of maximal packing fraction achieved by tapping the powders. With this parameter, one obtains the final packing fraction $\eta_{\infty}$ consequently the more common parameter, Hausner ratio $\mathrm{Hr}=\frac{\eta_{500}}{\eta_{0}}[6,40,41]$. The Hausner ratio is

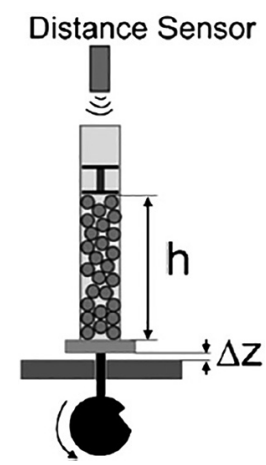

Fig. 2. Sketch of the GranuPack equipment [38]. The powder is placed in a metallic tube and a light hollow cylinder is placed on the top of the pile to keep it flat during the compaction process. The taps correspond to successive free falls of the tube over a distance $\Delta \mathrm{Z}$. After each tap, the height h of the pile is measured. Finally, a compaction curve is obtained. correlated to the powder flowability whereby high value of $\mathrm{Hr}$ corresponds to low flowability.

\subsection{GranuCharge}

Electrostatic charges are created due to the triboelectric effect inside a powder during a flow; meaning that the particles exchange charge at the result of contact with the other particles and devices wall. Fig. 3 illustrates GranuCharge equipment, it is a very useful equipment to measure the ability of a flowing powder to be charged electrostatically. It measures electrostatic charge of the powders by putting them in motion under the influence of gravity. In this measurement, the experimental set up consists of three parts: a stainless-steel pipe, a V-tube and a Faraday cup. The stainless-steel pipe is for feeding the $\mathrm{V}$-tube by automatic rotation inside the tube.

In general, the material of the $\mathrm{V}$-tube can be selected in the following list: stainless steel 316 L, aluminum 6063-T6, borosilicate glass, ABS, PVC and HDPE. The Stainless Steel $316 \mathrm{~L}$ was used in this study. The Vtube itself consists of two tubes with the length of $\mathrm{L}=350 \mathrm{~mm}$ and diameter $\mathrm{D}=47 \mathrm{~mm}$; the two parts are connected together with the angle of $90^{\circ}$. After feeding the tube by a stainless-steel pipe the powders flow inside V-tube and at the end they fall into a faraday cup which is connected to an electrometer to measure the powders charge obtained during flow. Before starting each test, the whole assembly has been connected to the earth to be sure that they are discharged. The electrometer is capable of measuring charge ranging $0.1 \mathrm{nC}-1 \mu \mathrm{C}$. The powder charge density is computed by dividing calculated charge by the mass of powder. The charge density unit is Coulomb per kilogram. The powders can obtain negative or positive charge after flow, based on their tendency to obtain or lose electron [15].

\subsection{Discovery HR3-rheometer}

The rheology of powders subject to vibration has been studied in this paper as well. With this objective, the Discovery HR3-rheometer has been implemented (see Fig. 4). The implemented geometry in this study is a cylindrical baffle cell (radius $=25 \mathrm{~mm}$, baffle height $=8.5$ $\mathrm{mm}$ ) with a four blades vane (radius $=7.5 \mathrm{~mm}$, length $=51 \mathrm{~mm}$ ). Only $21 \mathrm{~mm}$ of vane height has been immersed inside the powder. Indeed, in the case of immersing the whole vane inside the powder, the torque applied to shear the powder sample exceeds the limit of the apparatus. The utilized cell is similar to the cylindrical couette cell with the annular gap $=10 \mathrm{~mm}$. The characterization cell is attached to a shaker at the bottom with the computer controlled sinusoidal transmitted
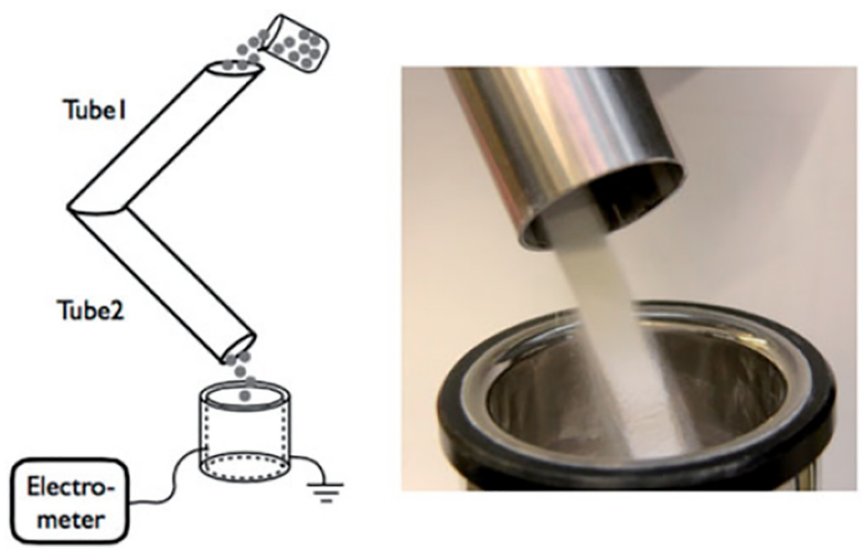

Fig. 3. Left: Sketch of the GranuCharge which is used to measure the electrostatic charge created inside a powder after flow in contact with a selected material. Right: Picture of the powder flowing from the tube 2 into the Faraday Cup measuring the electrostatic charge [42]. 


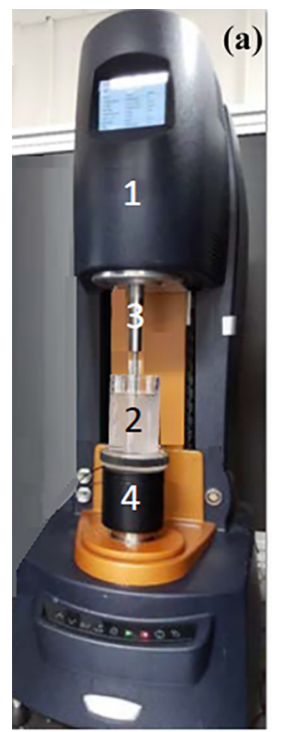

1. Rheometer «Discovery HR3 »

2. Measurement cell

3. Vane axis

4. Vibrator base

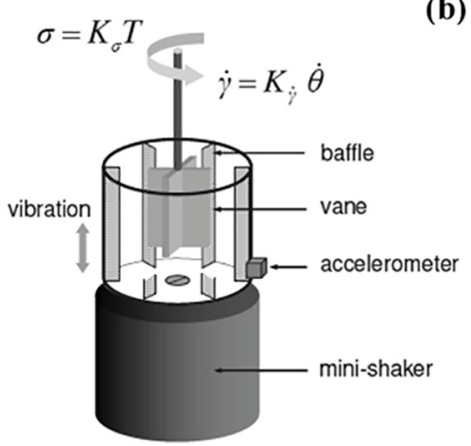

Fig. 4. (a) Rheometer discovery (b) Schematics of couette type cell connected to shaker [43]

vibration in close-loop system; detailed information can be found in $[44,45]$. The mechanical vibrational stress corresponds to the sample vibrational energy which is defined as $\sigma_{v}=(1 / 2) \rho A^{2}(2 \pi f)^{2}$, where $\rho$ is the powder density, A is the vibration amplitude and $f$ is the vibration frequency. The frequency and amplitude of vibration which has been used in this study is $60 \mathrm{~Hz}$ and $100 \mu \mathrm{m}$, respectively. Pre-vibration has been applied at $70 \mathrm{~Hz}$ during 10 min for removing any packing history before each measurement. The volume fraction of glass beads is $\phi_{\text {glass }} \approx 0.61[21,45]\left(\rho_{\text {glass }}=2500 \mathrm{~kg} / \mathrm{m}^{3}\right)$. All tests have been carried out at imposed shear stress with the range of 4.5-188 Pa and the measured shear rate ranged as $3 \times 10^{-5}-10 \mathrm{~s}^{-1}$.

\subsection{Humid control}

In this stage, the measurements were performed first with the GranuDrum and the GranuCharge with the objective of studying effect of humidity. The powder was placed in a rotating drum at $1 \mathrm{rpm}$ with a controlled humid air flow during $1 \mathrm{~h}$. As the powder was continuously mixed under drum rotation, the humidity absorption is assumed to be sufficient to observe changes in powder behavior. With this method, the accessible range for humidity is $\mathrm{RH}=35-95 \% \pm 5 \%$ inside the drum.

On the other side, another methodology has been utilized for the humid control of powders. In this case, the powder has been kept in a humid chamber longer to ensure absorption of enough humidity. With this purpose, the sufficient range of humid control time has been reported to be two days as minimum and one week as maximum storage time in a humid chamber for glass beads [46,47]. According to the literature the powder behavior has the same trend in both storage times. Therefore, here in our study each powder sample has been kept $72 \mathrm{~h}$ in a humid chamber and the range of imposed humid value was between $35 \%$ up to $90 \%$ at $20{ }^{\circ} \mathrm{C}$. The relative humidity of powders has been confirmed by using TH200 thermoconstanter (Novasina, Switzerland) at a temperature of $25{ }^{\circ} \mathrm{C}$; after taking them from the humid chamber. This apparatus has a measuring range comprised between 0.05 and 1.00 with an accuracy of \pm 0.01 over a temperature range between 0 and $50{ }^{\circ} \mathrm{C}$. More precisely, $1 \mathrm{~g}$ sample is introduced into a polypropylene cup deposited in the sealed enclosure of the apparatus. The free water moistens or dries the air inside the enclosure until the balance is reached. The relative humidity has been measured using an electrolytic sensor. After humid control then the powder characterization has been done by Discovery HR3-Rheometer under vibration.

\section{Results}

\subsection{Influence of surface treatment}

\subsubsection{Flowability}

In the literature, a vast body of researches conducted to study the powder flow behavior by different shear cell tests [48-53]. In our previous study we studied flowability of raw and hydrophobic glass beads with shear cell [31]. For this measurement, FT4 powder rheometer was utilized to evaluate powder flow at high shear rate by rotating shear head. In this measurement, first the powder was preconsolidated at $9 \mathrm{kPa}$ normal stress. Then, decreasing normal stresses from 7 to $3 \mathrm{kPa}$ by $1 \mathrm{kPa}$ steps were successively applied and the shear stresses $(\tau)$ (s) required to make the powder flow (i.e. to induce preconsolidated powder bed failure) were recorded. (see Fig. 5). In our measurements, the yield locus presented the same values for both powders over the entire range of normal stress showing similar flow behavior in raw and hydrophobic glass beads.

In addition, the flowing factor $f f$ characterizes powder flowability in this measurement. This factor is calculated by yield locus approach of software, whereby major principal stress $\left(\sigma_{1}\right)$ and unconfined yield strength $\left(\sigma_{c}\right)$ dedicated based on yield locus approach $\left(f f=\sigma_{1} / \sigma_{c}\right)$. The flowing factor has been recorded as $19.70 \pm 0.81$ and $19.74 \pm$ 1.66 for raw and hydrophobic glass beads, respectively.

According to the Jenike classification [54], powders are considered as not flowing for $f f<1$, very cohesive for $1<f f<2$, cohesive for $2<f f<4$, easy-flowing for $4<f f<10$ and free-flowing for $f f>10$. Therefore, based on this criteria our powders fall into category of free-flowing powders with the same flowability at high shear rate condition.

\subsubsection{Charge}

The electrostatic charge measurement of raw and hydrophobic glass beads has been performed with the GranuCharge equipment with the humidity and temperature range at $37-44 \%$ and $22{ }^{\circ} \mathrm{C}$, respectively. Fig. 6 represents the results corresponding to this test. Three values have been collected for each sample. These values include initial charge $\mathrm{q}_{0}$, final charge $\mathrm{q}_{f}$ and $\Delta \mathrm{q}=\mathrm{q}_{f}-\mathrm{q}_{0}$. The initial charge was measured before performing the test by directly introducing the powder inside Faraday cup and final charge is a measurement after powder flow through the V-tube. The obtained results show that both powders had almost zero initial electrostatic charge. After flow inside stainless steel V-tube, the raw glass beads kept this tendency for having almost zero charge

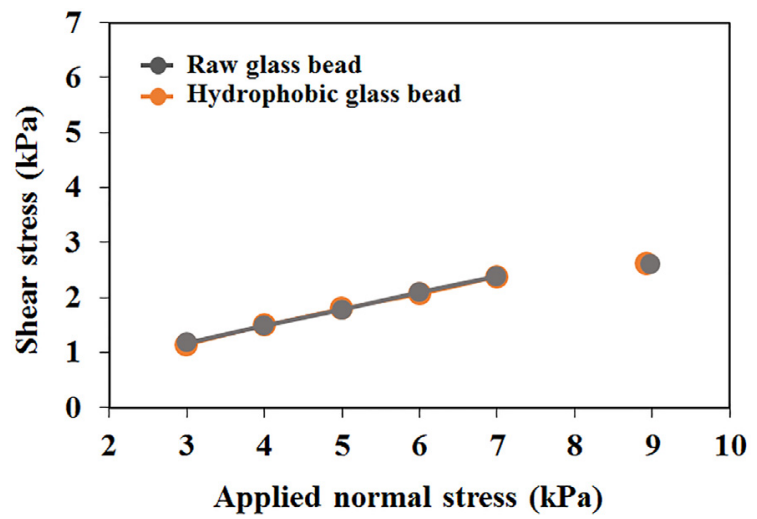

Fig. 5. Flow parameters derived from shear cell test for raw and hydrophobic glass beads [31]. Each test has been repeated three times and the measurements have been done in ambient temperature. Error bars represent standard errors; they are not visible as their size are inferior to the marker size. 


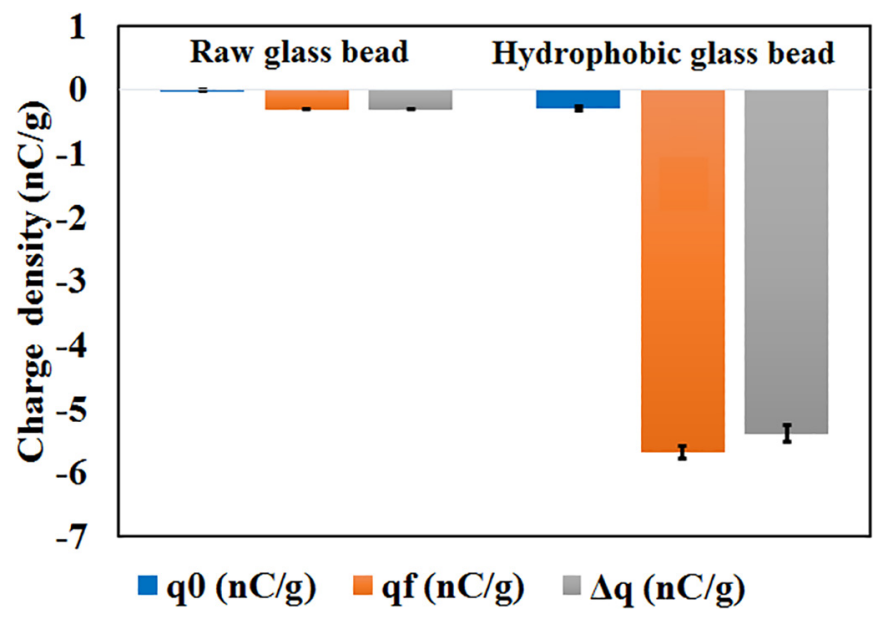

Fig. 6. Evolution of the electrostatic charge of raw and hydrophobic glass beads. $q_{0}$ is initial electrostatic charge before flow, $q_{f}$ is the final electrostatic charge after powder flow inside $\mathrm{V}$-tube and $\Delta \mathrm{q}$ is the difference between the initial and the final charge. Error bars represent standard errors (corresponding to three repeated measurements); some were not visible as their size was inferior to the marker size. The measurement performed by the GranuCharge equipment. The humidity and temperature range are 37-44\% and 22 ${ }^{\circ} \mathrm{C}$, respectively.

however hydrophobic glass beads obtained a negative charge. The quantity of each sample was $50 \mathrm{~mL}$ during measurement.

\subsubsection{Cohesion}

Fig. 7 shows the evolution of the cohesion $\sigma_{f}$ and the flowing angle $\alpha_{f}$ of raw and hydrophobic glass beads. It has been observed that cohesion of both samples have been enhanced by increasing the rotating speed of the drum, meaning that their flowability decreases under motion,

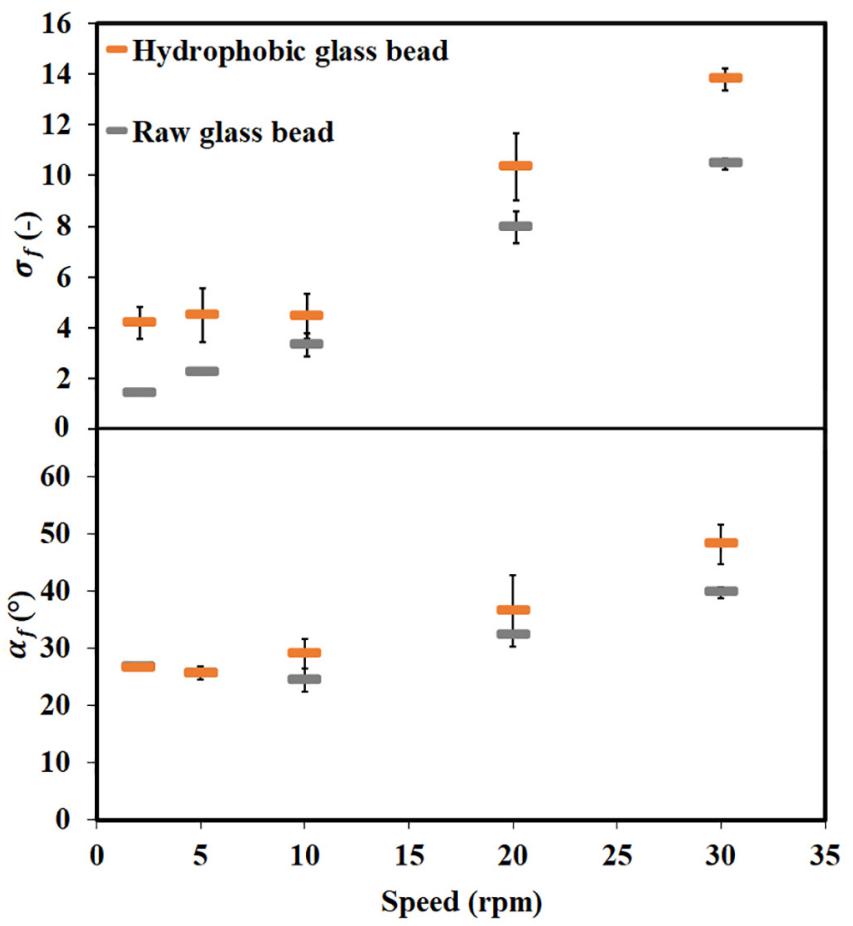

Fig. 7. Evolution of the flowing angle and cohesion of raw and hydrophobic glass beads under different rotational speed with the measurements performed by GranuDrum. Error bars represent standard errors (corresponding to three repeated measurements); some were not visible as their size was inferior to the marker size. The humidity and temperature range are $37-44 \%$ and $22{ }^{\circ} \mathrm{C}$, respectively. particularly after $10 \mathrm{rpm}$. Hydrophobic glass beads showed slightly more cohesion than raw glass beads. This could be explained by the GranuCharge measurements where hydrophobic glass beads presented more electrostatic charge than raw glass beads. This can lead to higher cohesion of hydrophobic glass beads under motion (see Fig. 6).

\subsubsection{Packing dynamics}

The compaction curves of raw and hydrophobic glass beads have been recorded with the GranuPack in standard conditions of temperature and pressure and they have been presented in Fig. 8. One observes the difference of density between two powders at the beginning and at the end of the compaction process. In addition, Fig. 9 presents the Hausner ratio $H_{r}$ where the packing density before tapping $\eta_{0}$, after $500 \operatorname{tap} \eta_{500}$ and the asymptotic packing fraction after an infinite number of tap $\eta_{\infty}$ obtained from the Eq. (1). One observes a slightly higher Hausner ratio for the hydrophobic glass beads than for the raw glass beads. This can be related to the cohesion since high Hausner ratio generally corresponds to poor flowability and therefore high cohesion. Also, the slight density difference of powders can be due to the different rearrangements of particles during measurement.

\subsection{Impact of humidity}

Influence of humidity on flowability of raw and hydrophobic glass beads has been studied. Indeed, it has been reported that humidity can change the charging of a powder [15] whereby it can induce variation of cohesion and consequently it modifies powder flowability. We implemented GranuDrum with the speed of $4 \mathrm{rpm}$ at standard conditions and GranuCharge equipments to measure powders cohesion and electrostatic charge under influence of humidity. Here, the humid control has been done with a rotating drum with humid-air controlled flow. The powders have been kept shortly $1 \mathrm{~h}$ in each selected humid range, the accessible range for humidity is $\mathrm{RH}=35-95 \% \pm 5 \%$ inside the drum.

Fig. 10 shows evolution of the cohesive index of raw and hydrophobic glass beads inside GranuDrum after humid control. The cohesive index of raw glass beads has been increased after $80 \%$ RH which shows high sensitivity of raw glass beads to the humidity after this value of humidity. However, the cohesive index of hydrophobic glass beads had almost constant value in all range of humid control, showing less sensitivity of this powder to the humidity. In addition, based on Fig. 11, we have seen that control glass beads have almost zero electrostatic charges in all ranges of humid control. While the electrostatic charge of hydrophobic glass beads decreased by increasing the

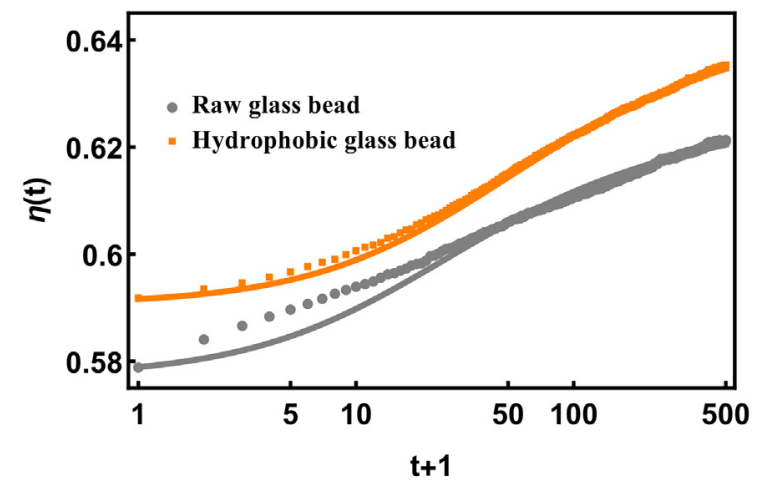

Fig. 8. Experimental compaction curves for respectively raw glass beads, represented by grey squares, and hydrophobic glass beads, represented by orange dots. Both curves have been fitted with the logarithmic model (Eq. (1)), represented by plain curves of the same color, in order to obtain the typical compaction time and the asymptotic packing fraction after an infinite number of tap. The humidity and temperature range are $37-44 \%$ and $22{ }^{\circ} \mathrm{C}$, respectively. 


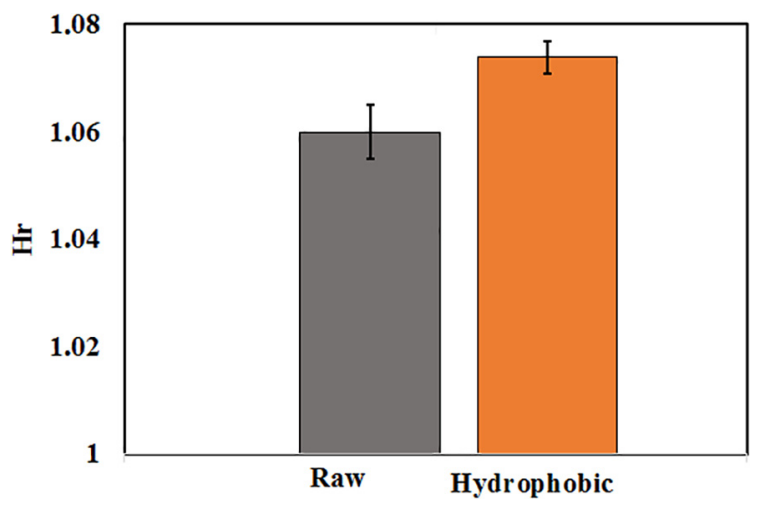

Fig. 9. Comparison between the Hausner ratio $\mathrm{Hr}=\frac{\eta_{500}}{\eta_{0}}$ of raw glass beads, in grey, and hydrophobic glass beads, in orange. Error bars represent standard errors (corresponding to three repeated measurements).

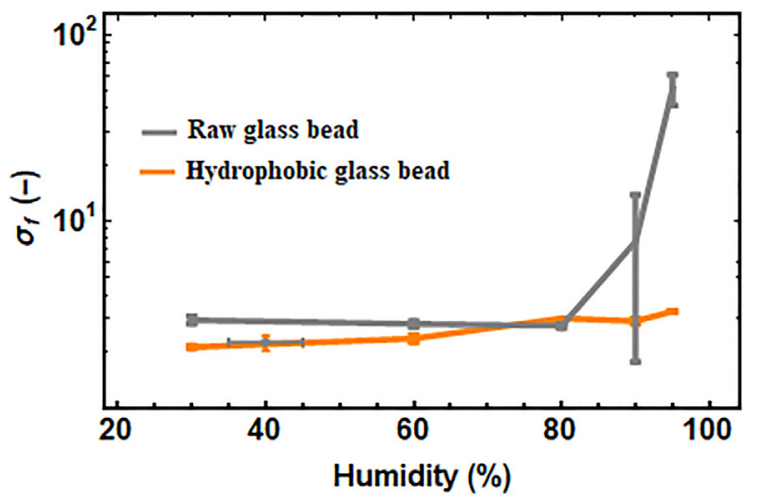

Fig. 10. Evolution of the cohesive index of raw and hydrophobic glass beads under humid control from $35 \%$ to $95 \% \pm 5 \%$. Error bars represent standard errors (correspond to three repeat of measurement); some were not visible as their size was inferior to the marker size. Both supplementary points out of the lines represent data from normal environmental conditions.

humidity up to $80 \%$ of humidity and after this range of humidity hydrophobic glass beads lost their electrostatic charge completely. Therefore, we have observed that the powder cohesion and electrostatic charge can evolve by humidity in spite of short humid control time. As a complementary test, the evolution of viscosity of our two formulations have been considered with Discovery HR3-Rheometer Discovery at low shear rate condition (Fig. 4). In this case, the powders humidity has been controlled longer during $72 \mathrm{~h}$ before each measurement in a humid chamber; then the rheological measurements have been done in an experimental room in ambient temperature and the humidity of room was not controlled during measurements.

Fig. 12 presents evolution of the viscosity of raw and hydrophobic glass beads as a function of humidity at $35-90 \% \mathrm{RH}$; the figure has been plotted in given shear rate at $\gamma=5 * 10^{-5} \mathrm{~S}^{-1}$. Based on viscosity curves, the hydrophobic glass beads presented higher viscosity than raw glass beads. This can be due to the fact that hydrophobic glass beads presented more electrostatic charge than raw glass beads in the humid range of 35-80\% (see Fig. 11). In addition, the viscosity curve of hydrophobic glass beads stayed always constant even after $80 \%$ of humidity. This is due to the tendency of hydrophobic glass beads to repel water even at $90 \%$ of humidity. By taking into account that the rheological measurements have been done in a dry room, then a slight amount of presented water in high humidity range on hydrophobic glass beads surface evaporated rapidly due to the tendency of this powder to repel the water. The rheological measurement time was between 20 and 30 min which was enough for evaporating the slight surface water and getting the powder recharged again. It should be indicated that the charge measurement time in Fig. 11 which has been done with GranuCharge was a few seconds, meaning that the powders did not have enough time to recharge.

In general, raw glass beads showed lower viscosity than the hydrophobic glass beads which can be due to the lower electrostatic charge of raw glass beads compared to hydrophobic glass beads. However, after $80 \%$ of humidity the viscosity of raw glass increased dramatically which is due to the sensitivity of raw glass beads to humidity that resulted in high cohesion. In addition, this increasing shift of viscosity of raw glass beads can be due to the condensation effect, however for the hydrophobic glass beads the viscosity was constant throughout the same range of humidity. It indicates that performing hydrophobic surface treatment on glass beads decreases the sensitivity of glass beads to the humidity therefore the viscosity of hydrophobic glass beads stayed constant in all range of humidity. In order to check the stability of hydrophobic surface formulation on glass beads, the contact angle measurement performed at the end of measurements performed by Discovery HR3-rheometer (the longest test in this paper).

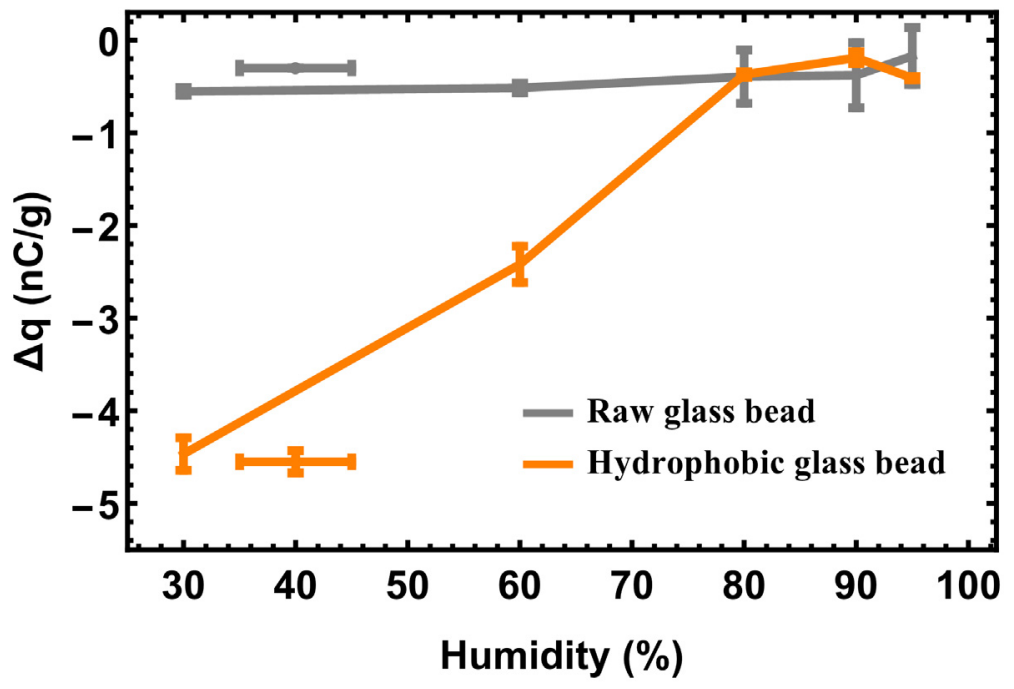

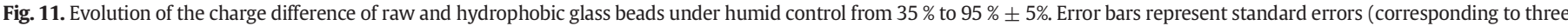

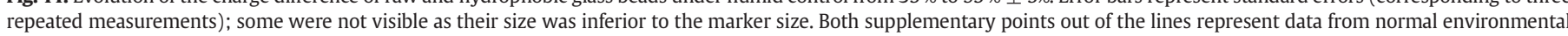
conditions. 


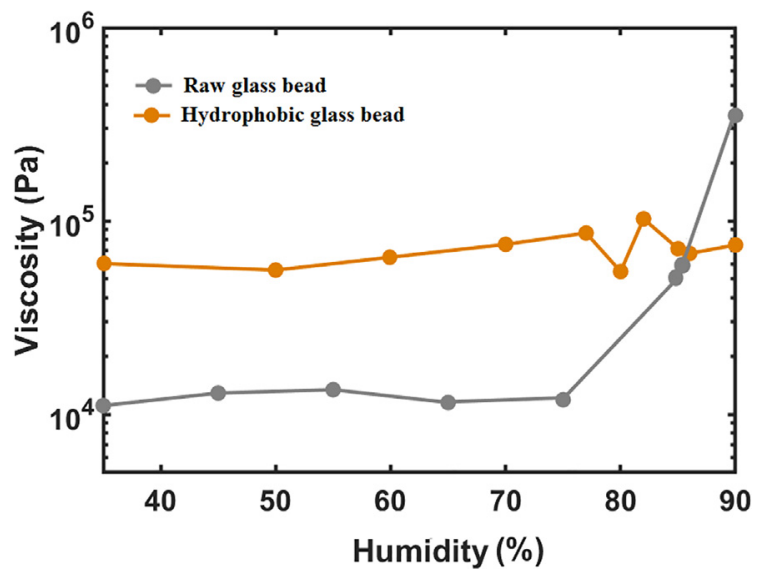

Fig. 12. Evolution of the viscosity of raw and hydrophobic glass beads under humid control from $35 \%$ to $90 \%$. The measurement has been done with Discovery HR3-rheometer under vibration and the figure has been plotted in a given shear rate $\gamma=5 * 10^{-5} s^{-1}$.

Hydrophobic glass beads presented the same water contact angle than before performing any test.

\section{Discussion}

The FT4 shear cell test results from our previous study [31] reported the same flowability for raw and hydrophobic glass beads at high shear rate condition. Whereby, these two powders classified as easy flowing powders based on Jenike classification [54]. These results are in agreement with the similar study performed on raw glass beads by modified shear apparatus [55], where dry raw glass beads recorded as non cohesive powder. In addition, they reported that the value of consolidation stress during measurement had no significant influence on the shear data $[50,55]$. According to the literature, the powder flowability was expected to increases when increasing particle size [31,56,57]. In fact, surface area to volume ratio decreases with increasing size which results in better flowability of large size powders.

In addition, based on GranuPack results one observes a small difference in cohesion between the two powders which is also observed with the Hausner ratio. Indeed a high Hausner ratio generally corresponds to a high cohesion. However this difference was not significant enough to observe a change in flowability as showed in Fig. 5. It should be mentioned that slightly higher value of the density in hydrophobic glass beads can be due to the different rearrangements of particles in this case.

According to the GranuDrum measurement, the powders cohesion was increasing by increasing speed of drum. This can be due to an internal effect such as more electrostatic charge exchange because of more contact time of particles inside drum. To verify this hypothesis the cohesion measurement has been done with GranuDrum at the speed of $30 \mathrm{rpm}$ with two different conditioning rotating times: $15 \mathrm{~s}$ as short rotating time and $30 \mathrm{~min}$ as long rotating time. The measurement has been performed as usual with 50 pictures separated by $0.5 \mathrm{~s}$. As illustrated in Fig. 13, it has been observed that the value of cohesion is higher when the rotating time is longer ( $30 \mathrm{~min}$ ). As explained already, this is due to the more charge exchange coming from more contact time, which it results in increasing electrostatic charge.

Discovery HR3-rheometer collected the data corresponding to vibrational rheology of powders after humid control. It has been observed that hydrophobic glass beads have higher viscosity than raw glass beads, which is consistent with the observations of the cohesion and the Hausner ratio. It has been reported that silanization (hydrophobic surface formulation) increases powder electrostatic charge [58] and consequently cohesion [59]. Here, hydrophobic glass beads presented more electrostatic charge than raw glass beads before applying

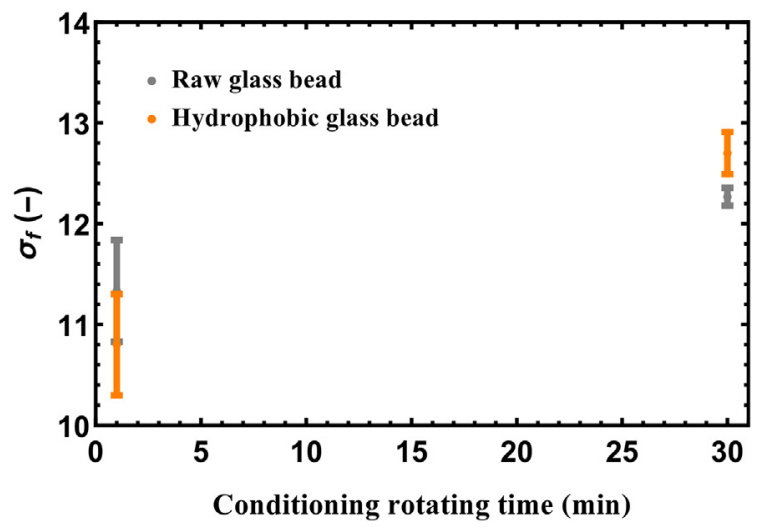

Fig. 13. Evolution of the cohesive index of raw and hydrophobic glass beads as a function of rotating time in GranuDrum. The rotating speed of drum was $30 \mathrm{rpm}$, error bars represent standard errors (corresponding to three repeated measurements). The humidity and temperature range are $37-44 \%$ and $22{ }^{\circ} \mathrm{C}$, respectively.

vibration, therefore applying vibration induced building up more electrostatic charge consequently higher viscosity has been recorded for hydrophobic glass beads. Based on literature, the particle electrostatic charge is in inverse relation with particles size, whereby by decreasing particles size the electrostatic charge per unit area increases which is due to the more specific surface area in small size particles compared to large particles [19]. Furthermore, according to the collected data with Discovery HR3-rheometer, we have seen that hydrophobic treatment decreases sensitivity of glass beads to the humidity since after $80 \%$ of humidity raw glass beads presented an impressive increase in the viscosity while viscosity of hydrophobic surface treated glass beads had almost constant value from the beginning up to end of humid control. This tendency of hydrophobic glass beads has been recorded with AFM measurement on single hydrophobic glass beads over the same range of humidity in the literature [27]. In our case, we collected the bulk behavior of hydrophobic glass beads over large relative humidity range which confirms the last findings on single hydrophobic glass beads [27]. In addition, it should be indicated that based on literature was found that there is a critical relative humidity at which the raw glass beads undergoes a transition from free-flowing to stick-slip behavior [26]. In our study, this critical value of humidity seems to be after $70 \%$ of relative humidity where raw glass beads presented a viscosity shift, this value is in agreement with the literature. In addition, this critical humidity increases with increasing particle size [26]. At the end of measurements performed by Discovery HR3rheometer (the most longest test in this paper) the contact angle measurement performed on hydrophobic glass beads with the objective of checking the stability of hydrophobic surface formulation on glass beads. Hydrophobic glass beads presented the same water contact angle than before performing any test. In the following the collected raw data from different set of measurements have been presented in Table 1.

\section{Conclusion}

This paper presented a study on flow behavior of raw and hydrophobic surface formulated glass beads. The objective was to evaluate the influence of hydrophobic surface treatment on glass beads as well as influence of humidity on flow behavior of raw and hydrophobic glass beads. With this objective, we considered our powders flow behavior under different processing dynamics to collect the essential factors and indices describing the behavior of a powder. Based on shear cell measurement, the powders reported similar flowability. However, GranuCharge measurement presented a charge difference between hydrophobic and raw glass beads. It reported negative charge for 
Table 1

Recapitulation of the different values measured with the set of instruments; the humidity and temperature range are $37-44 \%$ and $22{ }^{\circ} \mathrm{C}$, respectively. $\sigma_{f}$ and $\alpha_{f}$ are correspond to the Fig. 7.

\begin{tabular}{lll}
\hline Measures & Raw glass beads & Hydrophobic glass beads \\
\hline$f f(-)$ & $19.70 \pm 0.81$ & $19.74 \pm 1.66$ \\
$\Delta \mathrm{q}(\mathrm{nC} / \mathrm{g})$ & $-0.30 \pm 0.01$ & $-4.55 \pm 0.12$ \\
$\sigma_{f}$ (a.u.) $(30 \mathrm{rpm})$ & $10.46 \pm 0.21$ & $13.80 \pm 0.43$ \\
$\alpha_{f}$ (a.u.) $(30 \mathrm{rpm})$ & $39.73 \pm 0.10$ & $48.13 \pm 3.43$ \\
$\eta_{0}$ (a.u.) & $0.58 \pm 0.01$ & $0.59 \pm 0.01$ \\
$\eta_{500}$ (a.u.) & $0.62 \pm 0.01$ & $0.64 \pm 0.01$ \\
$\eta_{\infty}$ (a.u.) & $0.63 \pm 0.01$ & $0.66 \pm 0.01$ \\
$\mathrm{Hr}$ (a.u.) & $1.06 \pm 0.01$ & $1.07 \pm 0.01$ \\
\hline
\end{tabular}

hydrophobic glass beads and almost zero charge for raw glass beads which was expected to influence the powders flow behavior. This difference in charge could explain the slight difference in the Hausner ratio and cohesion. This issue has been confirmed with the cohesive index values measured by GranuDrum, the hydrophobic glass beads presented higher cohesion under rotation with GranuDrum which was indicative of decreasing flowability of hydrophobic glass beads under motion due to the tendency of this powder to build up electrostatic charge. In addition, the influence of air humidity on utilized powders has been studied with Discovery HR3-rheometer at low shear rate condition. The obtained results evidenced that under vibrational rheology the hydrophobic glass beads is less flowable than raw glass beads. It was because under vibration the electrostatic charge built up by hydrophobic glass beads that it was resulted in higher viscosity in this power. At high shear rate condition with shear cell test, both powders presented same flowability while at low shear rate condition with HR3-rheometer the raw glass beads showed lower flowability. This is because at high shear rate the powders are in frictional regime meaning that the friction between particle-particle governs the power flowability. Since raw and hydrophobic glass beads had similar surface properties therefore similar flowability has been recorded for them at frictional regime; however at low shear rate condition the flowability of powders are linked to their surface cohesion where the hydrophobic glass beads with more surface cohesion (due to the electrostatic charge) presented high viscosity. Furthermore, it has observed that by doing hydrophobic treatment on raw glass beads the sensitivity of powder to the humidity decreases, whereby even at high range of humidity it kept its flowability as in low humidity range. However, raw glass beads lost their flowability dramatically at higher value of humidity.

\section{Declaration of Competing Interest}

The authors declare that they have no known competing financial interests or personal relationships that could have appeared to influence the work reported in this paper.

\section{Acknowledgments}

This study is conducted in the framework of the "PowderReg" project, funded by the European program Interreg VA GR within the priority axis 4 "Strengthen the competitiveness and the attractiveness of the Grande Région / Großregion".

\section{References}

[1] E. Guerin, P. Tchoreloff, B. Leclerc, D. Tanguy, M. Deleuil, G. Couarraze, Rheological characterization of pharmaceutical powders using tap testing, shear cell and mercury porosimeter, Int. J. Pharm. 189 (1) (1999) 91-103.

[2] J. Xiang, L. Liu, X. Cui, Y. He, G. Zheng, C. Shi, Effect of Fuller-fine sand on rheological, drying shrinkage, and microstructural properties of metakaolin-based geopolymer grouting materials, Cem. Concr. Compos. 104 (2019) 103381.
[3] J.A. Grabowski, V.-D. Truong, C.R. Daubert, Nutritional and rheological characterization of spray dried sweetpotato powder, LWT Food Sci. Technol. 41 (2) (2008) 206-216.

[4] P. Coussot, Rheometry of Pastes, Suspensions, and Powders: Applications in Industry and Environment, John Wiley \& Sons, 2005.

[5] C. Salameh, J. Scher, J. Petit, C. Gaiani, C. Hosri, S. Banon, Physico-chemical and rheological properties of Lebanese kishk powder, a dried fermented milk-cereal mixture, Powder Technol. 292 (2016) 307-313.

[6] G. Lumay, F. Boschini, K. Traina, S. Bontempi, J.-C. Remy, R. Cloots, N. Vandewalle, Measuring the flowing properties of powders and grains, Powder Technol. 224 (2012) 19-27.

[7] U.V.Shah, V. Karde, C. Ghoroi, J.Y.Y. Heng, Influence of particle properties on powder bulk behaviour and processability, Powder Technol. 518 (1-2) (2017) 138-154.

[8] A. Castellanos, The relationship between attractive interparticle forces and bulk behavior in dry and uncharged fine powders, Adv. Phys. 54 (4) (2005) 263-376.

[9] A.M. Stoklosa, R.A. Lipasek, L.S. Taylor, L.J. Mauer, Effects of storage conditions, formulation, and particle size on moisture sorption and flowability of powders: A study of deliquescent ingredient blends, Food Res. Int. 49 (2) (2012) 783-791.

[10] European Pharmacopoeia 7.0, Powder Flow Chapter 2.9.36, 2010308.

[11] R.L. Carr, Evaluating flow properties of solids, Chem. Eng. 72 (1965) 163-168.

[12] H. Hausner, Friction conditions in a mass of metal powder, Int. J. Powder Metall. 3 (1967) 7-13.

[13] S. Wang, Z. Yunlong, Y.S. Zhou, S. Li, F. Fan, L. Lin, Z.L. Wang, Molecular surface functionalization to enhance the power output of triboelectric nanogenerators, J. Mater. Chem. A 4 (10) (2016) 3728-3734.

[14] F. Fulchini, U. Zafar, C. Hare, M. Ghadiri, H. Tantawy, H. Ahmadian, M. Poletto, Relationship between surface area coverage of flow-aids and flowability of cohesive particles, Powder Technol. 322 (2017) 417-427.

[15] A. Rescaglio, J. Schockmel, N. Vandewalle, G. Lumay, Combined effect of moisture and electrostatic charges on powder flow, EPJ Web of Conferences 140, EDP Sciences 2017, p. 13009.

[16] E. Mersch, G. Lumay, F. Boschini, S. Bontempi, N. Vandewalle, Effect of an electric field on an intermittent granular flow, Phys. Rev. E 81 (4) (2010), 0413097,

[17] D.A. Hays, J. Mason, P.J. Mason, R.E. Zeman, D. Jackson, Fabrication of 3D Objects Via Electrostatic Powder Deposition, US Patent App.15/050 2016729.

[18] T. Stichel, T. Brandl, T. Hauser, B. Geißler, S. Roth, Electrophotographic multimaterial powder deposition for additive manufacturing, Proc. CIRP 74 (3) (2018) 249-253.

[19] U. Zafar, F. Alfano, M. Ghadiri, Evaluation of a new dispersion technique for assessing triboelectric charging of powders, Int. J. Pharm. 543 (1-2) (2018) 151-159.

[20] E.M. Sloot, N.P. Kruyt, Theoretical and experimental study of the transport of powders by inclined vibratory conveyors, Powder Technol. 87 (3) (1996) 203-210.

[21] N. Gaudel, S. Kiesgen De Richter, N. Louvet, M. Jenny, S. Skali-Lami, Bulk and local rheology in a dense and vibrated granular suspension, Phys. Rev. E 96 (6) (2017), 062905,

[22] G. Lumay, K. Traina, F. Boschini, V. Delaval, A. Rescaglio, R. Cloots, N. Vandewalle, Effect of relative air humidity on the flowability of lactose powders, J. Drug Deliv. Sci. Technol. 35 (2016) 207-212.

[23] N. Vandewalle, G. Lumay, F. Ludewig, J.E. Fiscina, How relative humidity affects random packing experiments, Phys. Rev. E 85 (3) (2012), 031309,

[24] J.E. Fiscina, G. Lumay, F. Ludewig, N. Vandewalle, The compaction dynamics of wet granular assemblies, Phys. Rev. Lett. 105 (4) (2010), 048001,

[25] M.J. Colbert, M. Grandbois, N. Abatzoglou, Identification of inter-particular forces by atomic force microscopy and how they relate to powder rheological properties measured in shearing tests, Powder Technol. 284 (2015) 396-402.

[26] A.J. Forsyth, S. Hutton, M.J. Martin, Effect of cohesive interparticle force on the flow characteristics of granular material, Powder Technol. 126 (2) (2002) 150-154.

[27] R. Jones, H.M. Pollock, J.A.S. Cleaver, C.S. Hodges, Adhesion forces between glass and silicon surfaces in air studied by AFM: effects of relative humidity, particle size, roughness, and surface treatment, Langmuir 18 (21) (2002) 8045-8055.

[28] G. Landi, D. Barletta, P. Lettieri, P. Massimo, Flow properties of moisturized powders in a Couette fluidized bed rheometer, Int. J. Chem. React. Eng. 10 (1) (2012) $8045-8055$

[29] G. Landi, D. Barletta, P. Massimo, Modelling and experiments on the effect of air humidity on the flow properties of glass powders, Powder Technol. 207 (1-3) (2011) 437-443.

[30] E. Teunou, J.J. Fitzpatrick, Effect of relative humidity and temperature on food powder flowability, J. Food Eng. 42 (2) (1999) 109-116.

[31] S. Enferad, J. Petit, C. Gaiani, V. Falk, J. Burgain, S. Kiesgen De Richter, M. Jenny, Effect of particle size and formulation on powder rheology, Part. Sci. Technol. (2020) 1-9.

[32] Z. Kutelova, H. Mainka, K. Mader-Arndt, W. Hintz, J. Tomas, Functionalization and surface modification of spherical glass beads, 7th International Conference for Conveying and Handling of Particulate Solids, Friedrichshafen, Germany, 2011.

[33] N. Taberlet, R. Patrick, E.J. Hinch, S shape of a granular pile in a rotating drum, Phys. Rev. E 73 (5) (2006), 050301,

[34] X.Y. Liu, E. Specht, J. Mellmann, Experimental study of the lower and upper angles of repose of powders in rotating drums, Powder Technol. 154 (2-3) (2005) 125-131.

[35] J. Rajchenbach, Flow in powders: from discrete avalanches to continuous regime, Phys. Rev. Lett. 65 (18) (1990) 2221.

[36] H.H. Hausner, Friction Conditions in a Mass of Metal Powder, Polytechnic Inst. of Brooklyn. Univ. of California, Los Angeles, 1967.

[37] S. Chikosha, T.C. Shabalala, H.K. Chikwanda, Effect of particle morphology and size on roll compaction of Ti-based powders, Powder Technol. 264 (310-319) (2014)

[38] G. Lumay, F. Boschini, K. Traina, S. Bontempi, J.-C. Remy, R. Cloots, N. Vandewalle, Measuring the flowing properties of powders and grains, Powder Technol. 224 (2012) 19-27. 
[39] J.B. Knight, C.G. Fandrich, C.N. Lau, H.M. Jaeger, S.R. Nagel, Density relaxation in a vibrated granular material, Phys. Rev. E 51 (5) (1995) 3957-3963.

[40] K. Traina, R. Cloots, S. Bontempi, G. Lumay, N. Vandewalle, F. Boschini, Flow abilities of powders and powders evidenced from dynamical tap density measurement, Powder Technol. 235 (2013) 842-852.

[41] A. Saker, M.G. Cares-Pacheco, P. Marchal, V. Falk, Powders flowability assessment in granular compaction: what about the consistency of Hausner ratio? Powder Technol. 354 (2019) 52-63.

[42] A. Rescaglio, F. De Smet, A. Luc, G. Lumay, Tribo-electrification of pharmaceutical powder blends, Particul. Sci. Technol. 37 (2019) 1024-1031.

[43] N. Gaudel, Rhéologie et contrôle des écoulements de dispersions granulaires par l'application de vibrations, PhD thesis Université de Lorraine, 2018, 198301,

[44] Ph. Marchal, N. Smirani, L. Choplin, Rheology of dense-phase vibrated powders and molecular analogies, J. Rheol. 53 (1) (2009) 1-29.

[45] C. Hanotin, S. Kiesgen De Richter, Ph. Marchal, L.J. Michot, C. Baravian, Vibrationinduced liquefaction of granular suspensions, Phys. Rev. Lett. 108 (19) (2012), 198301, .

[46] L. Oger, C. El Tannoury, R. Delannay, Y. Le Gonidec, I. Ippolito, Y. Roht, I. GómezArriaran, Effect of grain size and material on humid granular avalanche events, Bulletin No. 123 of the Utah Engineering Experiment Station, hal-01825012v2, 2018.

[47] L. Oger, C. El Tannoury, R. Delannay, Y. Le Gonidec, I. Ippolito, Y.L. Roht, I. GómezArriaran, Dynamic behavior of humid granular avalanches: optical measurements to characterize the precursor activity, Phys. Rev. E 101 (2) (2020), 022902, .

[48] A. Angus, L.A.A. Yahia, R. Maione, M. Khala, C. Hare, A. Ozel, R. Ocone, Calibrating friction coefficients in discrete element method simulations with shear-cell experiments, Powder Technol. 372 (2020) 290-304.

[49] M. Lupo, D. Schütz, R. Maione, E. Riedl, D. Barletta, M. Poletto, Assessment of a powder rheometer equipped with a cylindrical impeller for the measurement of powder flow properties at low consolidation, Powder Technol. 357 (2019) 281-290.
[50] L.A.A. Yahia, T.M. Piepke, R. Barrett, A. Ozel, R. Oconeütz, R. Maione, E. Riedl, D. Barletta, M. Poletto, Development of a virtual Couette rheometer for aerated granular material, AIChE J. 66 (6) (2020), e16945, .

[51] K. Saleh, M.T.M. Abou Jaoude, M. Morgeneyer, E. Lefrancois, O. Le Bihan, J. Bouillard, Dust generation from powders: a characterization test based on stirred fluidization, Powder Technol. 255 (2014) 141-148.

[52] I. Tomasetta, D. Barletta, P. Lettieri, M. Poletto, The measurement of powder flow properties with a mechanically stirred aerated bed, Chem. Eng. Sci. 69 (1) (2012) 373-381.

[53] V. Francia, L.A.A. Yahia, R. Ocone, A. Ozel, From quasi-static to intermediate regimes in shear cell devices: theory and characterisation, KONA Powder Part. J. 2021018 (2021).

[54] A.W. Jenike, Storage and flow of solids, Bulletin No. 123 of the Utah Engineering Experiment Station 53, No. 26, November 1964, 1976.

[55] P. Pierrat, D.K. Agrawal, H.S. Caram, Effect of moisture on the yield locus of granular materials: theory of shift, Powder Technol. 99 (3) (1998) 220-227.

[56] K.K. Lam, J.M. Newton, Influence of particle size on the adhesion behaviour of powders, after application of an initial press-on force, Powder Technol. 73 (2) (1992) 117-1257.

[57] S. Enferad, Compactage et Vieillissement des Poudres: Influence de la Formulation, $\mathrm{PhD}$ thesis Université de Lorraine, 2020.

[58] K.W. Biegaj, M.G. Rowland, T.M. Lukas, J.Y.Y. Heng, Surface chemistry and humidity in powder electrostatics: a comparative study between tribocharging and corona discharge, Acs Omega 2 (4) (2017) 1576-1582.

[59] A. Hassanpour, M. Ghadiri, Characterisation of flowability of loosely compacted cohesive powders by indentation, Part. Part. Syst. Charact. 24 (2) (2007) 117-123. 